\title{
Thorax
}

\section{EXPLORING THE CHALLENGES OF ACCESSING MEDICATION FOR PATIENTS WITH CYSTIC FIBROSIS}

\begin{tabular}{|r|l|}
\hline Journal: & Thorax \\
\hline Manuscript ID & thoraxjnl-2021-217140.R2 \\
\hline Article Type: & Brief communication \\
\hline Duthor: & O2-Aug-2021 \\
\hline Complete List of Authors: & $\begin{array}{l}\text { Herbert, Sophie; University of Nottingham School of Medicine, Evidence } \\
\text { Based Child Health Group } \\
\text { Rowbotham, Nicola; University of Nottingham School of Medicine, } \\
\text { Evidence Based Child Health Group } \\
\text { Smith, Sherie; University of Nottingham School of Medicine, Evidence } \\
\text { Based Child Health Group } \\
\text { Wilson, Patrick; Nottingham University Hospitals NHS Trust, Pharmacy } \\
\text { Department } \\
\text { Elliott, Zoe; Parent of children with Cystic Fibrosis } \\
\text { Leighton, Paul; University of Nottingham, NIHR Research Design Service } \\
\text { for the East Midlands } \\
\text { Duff, Alistair; Leeds Teaching Hospitals NHS Trust, Clinical \& Health } \\
\text { Psychology; University of Leeds, Leeds Institute of Health Sciences } \\
\text { Smyth, Alan; University of Nottingham School of Medicine, Evidence } \\
\text { Based Child Health Group }\end{array}$ \\
\hline Keywords: & \begin{tabular}{l} 
Cystic Fibrosis, Psychology, Respiratory Infection \\
\hline
\end{tabular} \\
\hline
\end{tabular}

\section{SCHOLARONE ${ }^{\mathrm{m}}$ \\ Manuscripts}


I, the Submitting Author has the right to grant and does grant on behalf of all authors of the Work (as defined in the below author licence), an exclusive licence and/or a non-exclusive licence for contributions from authors who are: i) UK Crown employees; ii) where BMJ has agreed a CC-BY licence shall apply, and/or iii) in accordance with the terms applicable for US Federal Government officers or employees acting as part of their official duties; on a worldwide, perpetual, irrevocable, royalty-free basis to BMJ Publishing Group Ltd ("BMJ") its licensees and where the relevant Journal is co-owned by BMJ to the co-owners of the Journal, to publish the Work in this journal and any other BMJ products and to exploit all rights, as set out in our licence.

The Submitting Author accepts and understands that any supply made under these terms is made by BMJ to the Submitting Author unless you are acting as an employee on behalf of your employer or a postgraduate student of an affiliated institution which is paying any applicable article publishing charge ("APC") for Open Access articles. Where the Submitting Author wishes to make the Work available on an Open Access basis (and intends to pay the relevant APC), the terms of reuse of such Open Access shall be governed by a Creative Commons licence - details of these licences and which Creative Commons licence will apply to this Work are set out in our licence referred to above.

Other than as permitted in any relevant BMJ Author's Self Archiving Policies, I confirm this Work has not been accepted for publication elsewhere, is not being considered for publication elsewhere and does not duplicate material already published. I confirm all authors consent to publication of this Work and authorise the granting of this licence. 


\title{
EXPLORING THE CHALLENGES OF ACCESSING MEDICATION FOR \\ PATIENTS WITH CYSTIC FIBROSIS
}

Authors: Herbert $\mathrm{S}_{1}$, Rowbotham $\mathrm{NJ}_{1}$, Smith $\mathrm{S}_{1}$, Wilson $\mathrm{P}_{2}$, Elliott $\mathrm{Z}_{3}$, Leighton $\mathrm{P}_{4}$, Duff $\mathrm{AJA}_{5}$, Smyth $\mathrm{AR}_{1}$

\section{Institutions:}

1. Evidence Based Child Health Group, School of Medicine, E Floor East Block, Queens Medical Centre, Nottingham NG7 2UH

2. Pharmacy Department, Nottingham University Hospitals, Queens Medical Centre, Nottingham NG7 2UH

3. Parent of Children with CF, Nottingham, UK

4. School of Medicine, University of Nottingham, NG7 2NR.

5. Regional Paediatric CF Unit, Leeds Teaching Hospitals NHS Trust, Leeds, UK

Correspondence: Dr Nicola Rowbotham, Evidence Based Child Health Group, Division of Child Health, Obstetrics \& Gynaecology, Queens Medical Centre, Nottingham NG7 2UH, UK; Nicola.Rowbotham@nottingham.ac.uk

\begin{abstract}
Author Contributions:
$\mathrm{SH}, \mathrm{NR}, \mathrm{SS}$, AS were involved in concept, design, data collection, analysis and preparing the final manuscript. PW and ZE involved in the data collection, analysis and preparing the final manuscript, PL involved in analysis and preparing the final manuscript. AJAD involved in preparing the final manuscript.
\end{abstract}

Keywords: Cystic fibrosis, medication, treatment burden 
Acknowledgements: This work was funded by the UK Cystic Fibrosis Trust, and the University of Nottingham. N Rowbotham was an NIHR Academic Clinical Fellow at University of Nottingham. A Smyth is part of the National Institute for Health Research Nottingham Biomedical Research Centre

Ethical Approval: Faculty of Medicine \& Health Sciences Research Ethics Committee deemed this work not to require ethical approval on 30/4/2019.

\begin{abstract}
Abbreviations: CF: Cystic Fibrosis; CFTR: Cystic Fibrosis Transmembrane Conductance Regulator; GP: General Practitioner (Primary Care Doctor); JLA: James Lind Alliance; MDT: Multi-disciplinary Team; PSP: Priority Setting Partnership; PwCF: people with CF.
\end{abstract}

\begin{abstract}
(99 words)
Reducing treatment burden in cystic fibrosis $(\mathrm{CF})$ is the top research priority for patients and clinicians. Difficulty accessing medication is one aspect of treatment burden. We investigated this with an online survey available globally for CF patients and health care professionals.

Almost three quarters of CF patients in our survey report difficulty getting repeat prescriptions on time and most community pharmacists experience interrupted supplies of CF specific medications. These barriers affect emotional and physical health of people with CF. Two thirds of people with CF would like to get all their CF medication from one place, their CF centre.
\end{abstract}

\title{
Introduction
}


Reducing treatment burden for patients with $\mathrm{CF}$ was voted the number one research priority by people with $\mathrm{CF}$ (pwCF), their parents/carers and healthcare professionals in a James Lind Alliance Priority Setting Partnership. (1) 'Treatment burden' describes the increased workload associated with health care affecting individuals' physical and psychological wellbeing (2). The James Lind CF2 project further explored this research priority.

$\mathrm{CF}$ is a multisystem disease requiring complex treatment including specialist medications (e.g. inhaled antibiotics and CFTR modulators) and non-specialist drugs (e.g. bronchodilators and multivitamins), the latter being drugs which are used almost exclusively in the treatment of pwCF which are usually prescribed by the CF team. High treatment burden is common to many chronic diseases, resulting in reduced adherence, wasted medication, health deterioration and poorer quality of life. (3)(4) Treatment burden for pwCF is considerable most spend around two hours per day on treatment. (5) Accessing prescribed medications is an issue for patients with chronic diseases which contributes to treatment burden. (6) We aimed to review the obstacles pwCF encounter when accessing medications and to demonstrate insight from professionals who provide medications (pharmacists and primary care doctors).

\section{Methods}

The James Lind CF2 project explores in depth some of the research priorities identified by the James Lind Alliance priority setting partnership for CF. (1) It is supported by the UK National Institute for Health Research (NIHR) and the UK CF Trust. An online survey for pwCF was conducted generating free-text data on accessing medications (5). A further survey for primary care doctors and community pharmacists (with guidance from a focus group) was distributed using SurveyMonkey ${ }^{\mathrm{TM}}$ and was available for 4 weeks in June 2019. Both 
quantitative 'single answer' and qualitative 'free text' questions were incorporated. A final questionnaire to pwCF (July 2019) asked; 'should all medication be accessed from secondary care?'.

The questionnaires were not country-restricted and were promoted via social media. They were anonymous; no personal data were stored, or financial incentive given. All responses to the questionnaires were non-compulsory. Data were downloaded into Excel for quantitative analysis and into NVivo for qualitative analysis. Prior to commencing this work we published our protocol at:

\section{https://nottingham-repository.worktribe.com/output/2226944}

\section{Results}

James Lind CF2 question "Do you ever have difficulties getting the medications you need from your GP/Pharmacist/hospital/homecare?” found 76\% (241/317) of pwCF had difficulty accessing medication (5). The demographics of the participants have previously been published (5). Of the pwCF who had difficulty accessing medication, 65 participants expanded with a free text response following on from the quantitative question. Six recurring themes were identified. (Figure 1) Emotive language was used in free-text responses describing challenges that pwCF experienced in accessing their medication (Figure 2).

The healthcare professionals' survey yielded 256 responses, including 249 from the UK. Almost a third of respondents did not have pwCF in their cohort $(31 \% ; 78 / 249)$, with the majority having one or two pwCF 45\% (114/249). Primary care doctors accounted for $16 \%$ of 
the responses (40/249). Data on prescribing revealed that 39\% (13/33) lacked confidence when prescribing CF specialist medication and 50\% (17/34) had been asked to prescribe medication that should be prescribed by secondary care. Suggestions on how the CF centre can support primary care included education, ensuring up-to-date guidelines and having access to discuss care. Many pwCF did not see value from primary care doctors' input, and some primary care doctors felt that their role was 'limited as there is such good access to specialists'. However, others perceived their role to be caring for the pwCF outside of the CF condition, 'including mental health, contraception, and vaccinations'.

Community pharmacists describe their role as a medication dispenser to ensure continuous treatment and optimising adherence. Difficulties obtaining medication supplies are reported by $55 \%$ (52/94) of community pharmacists including: pancreatic enzyme replacement therapy; dornase alfa; CF specific vitamin preparations; and colistimethate sodium. 'Supply problems are a major issue, this is not a pharmacy problem but industrial pharmaceutical companies causing the delay'. Some specialist medications have a quota restricting supply, 'for example a manufacturer restricts supply to 2 per month, but you have a script for 12'. Expensive medications with short shelf-lives are not kept in stock because of the potential for waste if treatment is changed. For routine repeat-prescriptions, $48 \%(95 / 196)$ of pharmacies require 1-2 days' notice. For specialist medication 39\% (75/196) of pharmacies require 3-4 days' notice with 10\% (19/196) requiring 1-2 weeks' notice. Only 40\% (76/192) of these have online repeat prescriptions requests.

PwCF and health care professionals were asked if all medication should be prescribed and accessed in secondary care (Figure 3). Overall, 65\% (25/40) pwCF wanted to access 
medication from secondary care. Reservations included travel distance, car parking and hospital pharmacy delays.

\section{Discussion}

We present a complex process undertaken by pwCF to access medication, including $76 \%$ of those surveyed expressing difficulty getting repeat prescriptions from primary care. Our data suggest that this is part of the high treatment burden impacting upon physical and mental health.

Although each group interviewed has specific difficulties and frustrations, all are agreed on the need to simplify the process. Research shows that patients with chronic diseases have difficulty accessing medication, issues including pharmacy stock, medication errors and short durations of prescriptions (7) - all issues raised in our study. This is exacerbated by the number of medications pwCF take (average 8 daily) (8).

Primary care doctors and community pharmacists describe the barriers to accessing medication including practitioners' lack of familiarity with specialist medication and interrupted supplies. A guide to improving the logistics of obtaining medicines in the community offers pwCF and their families, practical suggestions on how to reduce such difficulties (9). Primary care doctors suggested that better communication with the CF centre and accessible guidelines would support their prescribing. The UK National Health Service (NHS) long-term plan (2019) aims for electronic prescribing (10) which may improve communication between the CF centre and primary care. 
Our study has limitations. Respondents were self-selected and those with negative experiences might be more motivated to participate. We did not survey CF centres. Not every respondent answered every question. The CF patient cohort responding to 'Should all medication be accessed from secondary care?' was a smaller group than the James Lind CF2 initial survey. We did not enquire as to whether a delivery service would aid access to medications. The surveys were conducted prior to the COVID-19 pandemic which might have altered responses, if patients were apprehensive about the infection risk in attending hospital. The strengths include the global reach and large number of responses to our original survey (941 responses from 21 countries) (1).

\section{Conclusion}

Accessing medication is a problem for pwCF adding to the high treatment burden, impacting upon both the emotional and physical health. The next step is for targeted quality improvement plans to help reduce treatment burden.

\section{References}

1. Rowbotham NJ, Smith S, Leighton PA, Rayner OC, Gathercole K, Elliott ZC, et al. The top 10 research priorities in cystic fibrosis developed by a partnership between people with CF and healthcare providers. Thorax. 2018;73(4):388-90.

2. Alsadah A, van Merode T, Alshammari R, Kleijnen J. A systematic literature review looking for the definition of treatment burden. Heliyon. 2020;6(4):e03641. doi:10.1016/j.heliyon.2020.e03641

3. Mair FS, May CR. Thinking about the burden of treatment. BMJ. 2014;349:g6680.

4. Sawicki GS, Sellers DE, Robinson WM. High treatment burden in adults with cystic fibrosis: challenges to disease self-management. J Cyst Fibros 2009;8(2):91-6. 
5. Davies G, Rowbotham NJ, Smith S, Elliot ZC, Gathercole K, Rayner O, et al. Characterising burden of treatment in cystic fibrosis to identify priority areas for clinical trials. J Cyst Fibros 2019:499-502 DOI 10.1016/j.jcf.2019.10.025

6. Lowton K, Ballard KD. Adult cystic fibrosis patients' experiences of primary care consultations: a qualitative study. Br J Gen Pract. 2006 Jul;56(528):518-25.

7. Wilson PM, Kataria N, McNeilly E. Patient and carer experience of obtaining regular prescribed medication for chronic disease in the English National Health Service: a qualitative study. BMC Health Serv Res. 2013;13:192. doi:10.1186/1472-6963-13192.

8. Sawicki, GS, Tiddens H. Managing treatment complexity in cystic fibrosis: Challenges and opportunities. Pediatr. Pulmonol. 2012;47: 523-533.

9. Duff AJ, Havermans T, Smith B. Improving self-management and adherence through interaction between the multidisciplinary team, the patient, and their families. In Sian Bentley, Carlo Castellani, Daniel Peckham, Nicola Shaw (Eds), Optimizing Pharmaceutical Care in Cystic Fibrosis. European Cystic Fibrosis Society. 2020; pp 233-249.

10. NHS. The NHS long term plan. 2019. https://www.longtermplan.nhs.uk/. Accessed October 2019.

\section{Conflicts of interest:}

AS reports a research grant and personal fees from Vertex. He has spoken at meetings supported by Teva and Novartis. These activities are outside the submitted work. In addition, Prof. Smyth has a patent alkyl quinolones as biomarkers of pseudomonas aeruginosa infection and uses thereof issued.

AD reports personal fees from Gilead, Chiesi, Novartis, ALK Abello and has attended dinners supported by Gilead and Chiesi, all outside the submitted work. 
Figure 1. Quotations from pwCF and their families for each theme identified. GP= General Practitioner (Primary care doctor)

$189 \times 178 \mathrm{~mm}(157 \times 157 \mathrm{DPI})$ 


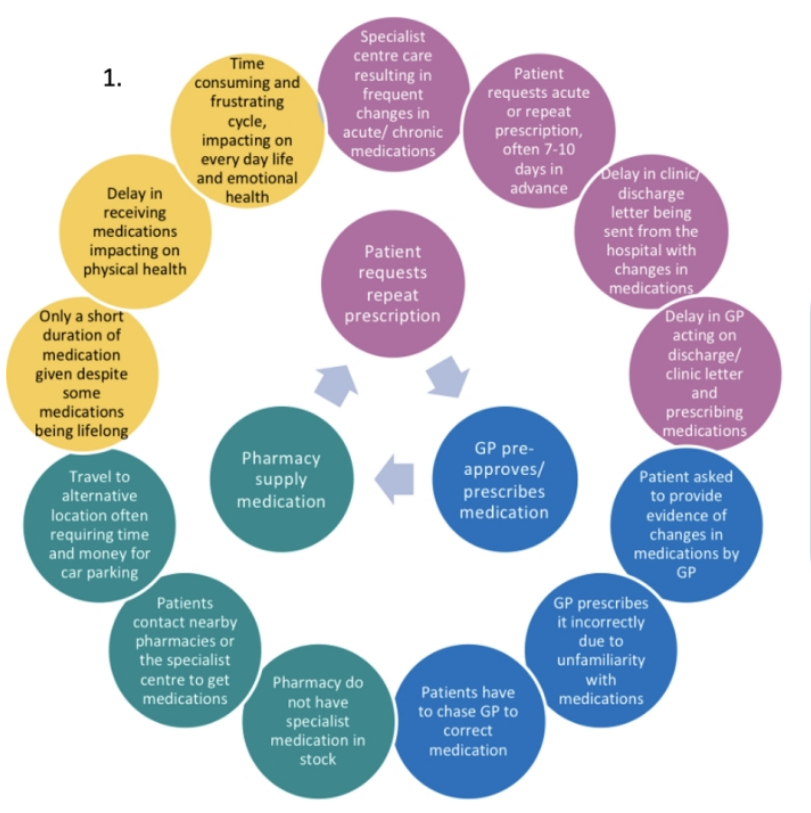

2.

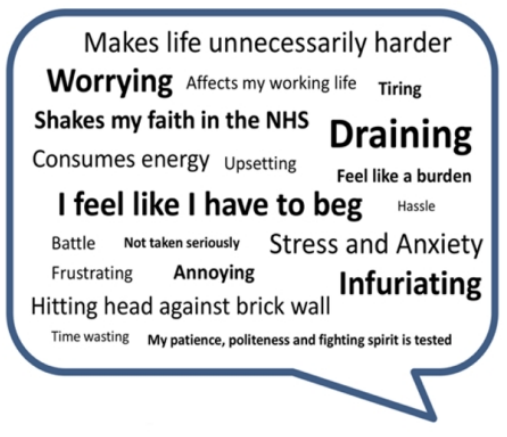

Figure 2. 1. Inner flow chart depicts the journey to accessing medications where the patient does not have a chronic, multisystem disease. The outer circle is a pathway described by the CF patient group that they take to access medications 2. Emotive language used by the patient group to describe the cycle in 1.

$97 \times 62 \mathrm{~mm}(400 \times 400 \mathrm{DPI})$ 


\section{Should all CF medications be accessed via secondary care?}

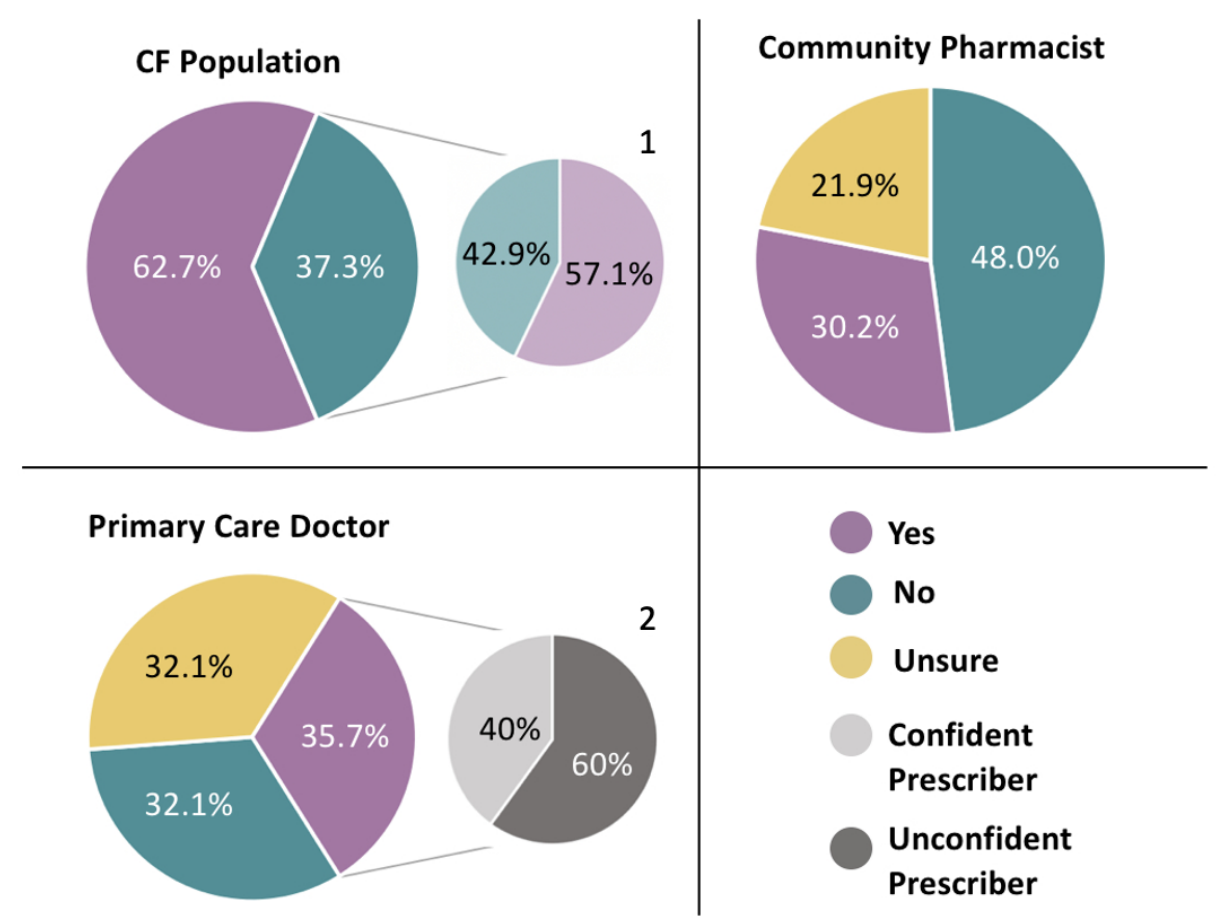

Figure 3. Should all medications be accessed from secondary care? Responses from the CF population,

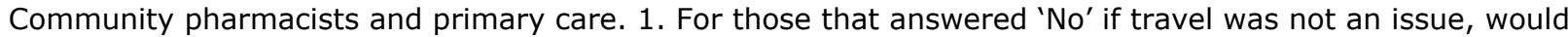
they change their mind? 2. For primary care doctors who said ' $N o^{\prime}$ ', how many were confident at prescribing

$$
73 \times 59 \mathrm{~mm}(400 \times 400 \text { DPI) }
$$

